\title{
Formation and breakup of compound pendant drops at the tip of a capillary and its effect on upstream velocity fluctuations
}

\author{
Zhizhao Che $^{a}$, Teck Neng Wong ${ }^{a,}$, Nam-Trung Nguyen ${ }^{a}, J_{. C .}$ Chai $^{b}$ \\ ${ }^{a}$ School of Mechanical and Aerospace Engineering, Nanyang Technological University, \\ 50 Nanyang Avenue, Singapore 639798, Singapore \\ ${ }^{b}$ Department of Mechanical Engineering, The Petroleum Institute, P.O. Box 2533, Abu \\ Dhabi, United Arab Emirates \\ * Corresponding author. Tel.: +65 67905587; fax: +65 67911859. \\ E-mail address:mtnwong@ntu.edu.sg.
}

\begin{abstract}
In this paper, the formation and breakup process of compound pendant drops (CPDs, pendant drops with smaller drops or bubbles in them) at the tip of a glass capillary and its effect on upstream velocity fluctuation are experimentally investigated. The formation process of an air/water compound drop from a CPD consists of four main stages. First, an air plug in the capillary flows into the small liquid pendant drop to initialize a small CPD. Next, a liquid slug flows into the CPD, and the liquid in the CPD accumulates. Subsequently, an air plug flows into the CPD, and it coalesces with the existing air bubble in the CPD. The accumulation and coalescence stages repeat, until the CPD reaches a critical weight, then the CPD finally breaks up to produce a compound drop. For the air/SDS-solution system, the bubbles in the CPDs do not coalesce, and the contact line of the CPDs initially climbs along the capillary and then moves downwards with the growth of the CPDs. The upstream velocity fluctuates during the periodical formation and breakup of the CPD due to Laplace pressure variation at the tip of the glass capillary. By adding surfactant into water, the fluctuation of the upstream velocity decreases. The size distribution of the compound
\end{abstract}


drops produced by the breakup of CPDs is quantified, and the results show that the current system is able to produce monodisperse compound drops.

\section{Keywords:}

Compound pendant drop, Breakup, Velocity fluctuation, Compound drops, Dropletbased microfluidics

\section{Introduction}

The formation and breakup of drops from the tip of a capillary is of great importance in many applications such as ink-jet printing [1], spraying, and food processing [2]. Moreover, they are of great scientific interest due to the richness of the underlying physics. The process is determined by the interplay of gravitational force, inertial force, viscous force, and surface tension force. When the flow rate in the capillary is small, the breakup of drops at the tip is in a dripping regime. When the flow rate is sufficiently large, dripping gives way to jetting [3], during which drops break up at the ends of long columns of liquid far downstream of the capillary tip [4]. In the dripping regime, the process of formation and breakup includes two main stages $[5,6]$. In the first stage, the pendant drop slowly grows in size, and the pendant drop is quasi-steady at every instant. The inertial force is negligible, and the flow is dominated by the surface tension force and the gravitational force. The shape of the pendant drop is determined by the balance of the surface tension force and the gravitational force. As the size of the pendant drop increases, it gradually reaches a critical size, at which the surface tension force cannot support the pendant drop, and then the process goes into the second stages. During the second stage, the neck of the pendant drop shrinks and less weight can be supported by the surface tension. Consequently, Plateau-Rayleigh instability [7] suddenly accelerates breakup and the drop finally detaches. The process of the growth and breakup of pendant drops repeats, and drops are produced periodically at the tip of the capillary.

The growth and breakup of pendant drops exhibits many interesting behaviors, 
and underlying physics can be uncovered by studying these behaviors [8]. Before the detachment of a drop from the capillary, a liquid thread is formed connecting the to-beformed drop and the remnant fluid at the tip. Experimental observations revealed that the breakup of the liquid thread shows a self-similar behavior during the final stage of the pinch-off process [2,9]. By considering the pinch-off of up to several hundreds of drops, rather than studying the drop growth behavior of a single drop, Ambravaneswaran et al. [10] studied the nonlinear response of drops. Their results showed that the time interval of dripping exhibits bifurcation and hysteresis from the time return map and the bifurcation diagram.

The growth and pinch-off process of the pendant drops is determined by the interplay of gravitational force, inertial force, viscous force, and surface tension force, which forms dimensionless groups of the Bond number, the capillary number, and the Weber number $[11,12]$. Surface tension is a significant factor affecting the growth and breakup of pendant drops. The surface tension of a liquid can be reduced by adding surfactant into the liquid, and the process of the growth and breakup of pendant drops can be altered [13-15]. The breakup process can also be manipulated by heat which induces surface tension gradient and Marangoni stress along the surface of the pendant drop [16], or alternatively by an electric field, which can deform the pendant drop and facilitate the breakup [17], or even create a tip-streaming phenomenon under a strong electric field [18].

A compound drop is a drop containing smaller drops or bubbles in it [19]. Compound drops have many promising applications in pharmaceutical and food industry, as well as waste water management. The behaviors of compound drops have been studied in various flow conditions, such as moving in quiescent flow [19], impact on flat surfaces [20], flow under thermocapillary force [21,22], formation in microcapillaries [23] and in microchannels [24], synthesis of polymeric microparticles $[25,26]$. However, to the best of our knowledge, compound pendant drops (CPDs), pendant drops with smaller drops or bubbles in them, have never been studied before. The combination of the pendant drop with the compound drop will result in many 
distinctive flow phenomena, and may trigger novel applications in chemical engineering, food processing industry, and pharmaceutical industry. For example, the capillary can be used as a tool to transfer or deliver individual compound drops by hanging the compound pendant drops at the capillary tip with the inner fluid being intact. The outer phase of the compound pendant drops can serve as vials for reactions or syntheses in the inner fluid, in which the processes of reactions or syntheses can be controlled individually and accurately. The outer phase can also be used as a protective layer for the inner phase, such as in food additives, drugs, or personal care products. If the inner phase is volatile and/or harmful to the environment, the outer phase can also minimize the mass transfer from the inner phase to the surrounding fluid.

For the formation of a simple or compound pendant drop at the tip of a capillary, the small size of the pendant drop produces a large Laplace pressure. During the formation and breakup, the simple or compound pendant drop causes large variation of Laplace pressure, and consequently, upstream velocity fluctuation. The variation of fluid velocity upstream can be welcome or unwelcome, depending on the application. A stable flow is required in applications such as detection in capillaries or microchannels. In order to get accurate results, the time for the samples to pass through the detection zone in capillaries or microchannels should be controlled in an accurate manner [27,28]. For reaction applications, the reaction time is a key factor that affects the products and should be accurately controlled [29]. Therefore, fluctuation of velocity should be avoided in these applications. On the contrary, velocity variation is preferred in some other applications, such as in micromixing [30-32]. The perturbation of velocity oscillation can enhance the mixing process by twisting the trajectory of the fluid particles and by stretching and folding the fluid elements [33,34]. van Steijn et al. [35] studied the velocity fluctuation of gas-liquid segmented flow in a microchannel, and they found that the velocity variation could be up to $30 \%$ of the mean velocity with a periodicity equivalent to the drop production process. Beer et al. [36] confirmed this effect for liquid-liquid phase aqueous drop production in oil. However, to the best of our knowledge, the velocity 
fluctuation produced by formation and breakup of simple and compound pendant drops has not been studied.

In this study, we investigated the formation and breakup process of CPDs at a vertical tip of a capillary and its effect on upstream velocity fluctuation. This paper is organized as follows. Firstly, experimental details including the experimental setup, the properties of the fluids, and the flow visualization method are described in Section 2. Then, the experimental results are provided and analyzed in Section 3. The stages of formation and breakup of CPDs are analyzed from sequences of images. The velocity fluctuation caused by the formation and breakup of CPDs is studied, and the effect of surfactant is investigated.

\section{Experimental}

\subsection{Formation of compound pendant drops}

The experimental setup is shown schematically in Fig. 1a. To form CPDs at the tip of a capillary, air-plug/water-slug flow is generated using a horizontal flow focusing geometry in a poly-methylmethacrylate (PMMA) channel [37,38]. A glass capillary is connected to the outlet of the microchannel perpendicularly to the PMMA channel and positioned downwards. The air plugs and the water slugs flow in the horizontal PMMA flow channel (Fig. 1b), subsequently flow into the glass capillary, and finally leave the capillary in a form of CPDs at the tip of the capillary (Fig. 1c).

The flow focusing geometry (Fig. 1b) is fabricated on a cast PMMA sheet with a thickness of $1 \mathrm{~mm}$ (Ying Kwang Acrylic Trading, Singapore). Three layers of PMMA were cut by $\mathrm{CO}_{2}$ laser cutting platform (Universal M-300, USA). They were subsequently aligned and bounded. At the outlet of the PMMA flow channel, a glass capillary (Sigma-Aldrich P0674, USA) was connected perpendicularly in the downward direction. The inner diameter $\left(\mathrm{D}_{i}\right)$ and the outer diameter $\left(\mathrm{D}_{o}\right)$ of the capillary are $0.5 \mathrm{~mm}$ and $1 \mathrm{~mm}$, respectively. The glass capillary was cut to a length 
of $91 \mathrm{~mm}$.

To avoid the breakup of bubbles at the connecting point between the PMMA microchannel and the glass capillary, a step structure was fabricated on the PMMA microchannel, as shown in Fig. 1d. The step structure is of a circular shape with the inner/outer diameters being the same as the inner/outer diameters of the glass capillary, which facilitates the alignment between the microchannel and the glass capillary, and ensures a smooth fluid flow to the glass capillary. Epoxy was applied at the corner to permanently connect the PMMA channel and the glass capillary. An image of the microchannel with the capillary tip being connected is shown in Fig. 1e.

To form air plugs in the PMMA microchannel, air was used as the dispersed phase and was introduced by a constant pressure through a water column of $14 \mathrm{~cm}$. DI water with/without surfactant sodium dodecyl sulfate (SDS) (Sigma-Aldrich L4509, USA) was used as the continuous phase, and was introduced by a syringe pump (KD scientific, USA). The properties of air, DI water, and the SDS solution (2\% by weight) are listed in Table 1. The surface tension of the SDS solution was measured using the pendant drop method by an FTA 200 system (First Ten Ångstroms, USA).

\subsection{Image acquisition and processing}

The flow in the glass capillary was recorded by a CCD camera (PULNiX TM6701AN, USA). The frame rate of the CCD camera was 30 frames per second (fps) during the experiment. A lens with a focal length of $16 \mathrm{~mm}$ (TAMRON, Japan) was mounted on the CCD. A white plate was used to provide a clear background for the CCD image. We used a halogen lamp and shined the light on the background to provide the illumination for the image acquisition. To get the details of the formation and breakup of the gas-liquid CPDs at the outlet of the capillary, we used a macro lens with magnification of $0.3 \times$ to $1 \times$ (Computar, USA). 
The image sequences of the air-plug/water-slug flow in the glass capillary was exported and processed by a customized MAT-LAB program. The velocity of the fluids was obtained by comparing the positions of the bubble fronts/rears between every two successive frames of the video. The image processing procedure is illustrated in Fig. 2 and listed as follows: (a) Read the video file. (b) Crop the region of the capillary. (c) Average many frames to get the background image. (d) Subtract the background image from each frame to obtain the bubbles in the images, and increase the contrast. (e) Convert the grayscale images to black-and-white binary images. (f) Take the average of the brightness. (g) Take the gradient of the blackand-white binary images to find out the location of the fronts and rears of the bubbles. (h) Calculate the velocities of the fronts and the rears of the air plugs by comparing two successive frames. Average the velocities of the fronts and the rears of the air plugs and take the average as the velocity of the fluids at that time. They are calculated as follows,

$u\left(t_{k}\right)=\frac{1}{N} \sum_{i=1}^{N} \frac{\Delta x_{i}}{t_{k+1}-t_{k}}$

where $t_{k}$ and $t_{k+1}$ are the time for the two successive frames, $\Delta x_{i}$ is the displacement of the fronts/rears of the air plugs between the two successive frames, $N$ is the number of fronts and rears of the air plugs in the observing window.

\section{Results and discussion}

\subsection{Formation and breakup of CPDs for air/water system}

The formation and breakup of CPDs at the tip of a vertical capillary shares some common features with that of simple pendant drops. However, due to the presence of the gas bubbles, the formation and breakup of CPDs is more complex, and it has many distinctive features different from the simple pendant drops. To study the dynamics of CPDs, a high speed camera (Fastec HiSpec 1, Fastec Imaging, USA) and a macro lens with magnification of $0.3 \times$ to $1 \times($ Computar, Japan) was used 
to focus at the tip of the glass capillary.

The typical stages in time sequence of the formation and breakup of a CPD are summarized as follows. After the breakup of the previous CPD, a small amount of liquid is left at the tip of the capillary, which forms a small pendant drop. (I) Initialization: Firstly, an air plug in the capillary flows into the small liquid pendant drop to initialize a small CPD, as shown in Fig. 3. (II) Accumulation: A liquid slug flows into the CPD, and the liquid in the CPD accumulates. The CPD increases in weight and in size, as shown in Fig. 4. (III) Coalescence: An air plug flows into the CPD, and it coalesces with the existing air bubble in the CPD. The CPD increases in size, as shown in Fig. 5. (IV) Breakup: Stages II and III repeat, until the CPD reaches a critical weight, then the CPD finally breaks up to produce a compound drop, as shown in Fig. 6. The four stages repeat and CPDs are formed and breaks up periodically. Each stage is analyzed in detail in the following subsections.

\subsubsection{Initialization of a small compound pendant drop}

After the breakup of the previous CPD, a small liquid pendant drop is left at the tip of the glass capillary. Due to the small size of the liquid pendant drop, the gravitational force is negligible. Hence, the pendant drop is almost spherical, as shown in Fig. 3a. As the first air bubble flows into the pendant drop, it experiences a scenario that part of the bubble is in the capillary and the rest is in the pendant drop. This is shown in Fig. $3 b-h$, while the schematic illustration is shown in Fig. 3j. The part in the pendant drop is almost spherical due to the small size of the bubble. As the whole air plug enters into the pendant drop, it relaxes from plug shape to spherical shape due to the effect of surface tension, as shown in Fig. 3i. This step marks the end of Stage I and the beginning of Stage II.

\subsubsection{Accumulation of liquid in the compound pendant drop}

After an air bubble relaxes to a spherical shape from Stage I in the CPD, the flow 
of water into the CPD is affected by the presence of the air bubble. Due to gravity, water is accumulated at the bottom, while the air bubble remains at the top of the CPD, as shown in Fig. 4a. The air bubble partially blocks the outlet of the capillary. After flowing out of the capillary, water flows to the bottom of the CPD through the thin liquid film on the side of the bubble, as shown in the image sequence in Fig. $4 \mathrm{a}-\mathrm{f}$, while the schematic illustration is shown in Fig. $4 \mathrm{~g}$. It was observed that the bubble on the axis of the capillary centerline is unstable, as it experiences the pushing force by the water flowing from the capillary. Consequently, the water pushes the bubble off the axis to reduce flow resistance, which causes the CPD to vibrate.

\subsubsection{Coalescence of bubble with the subsequent air plug}

After a liquid slug flows into the CPD, an air plug flows out of the capillary to coalesce with the bubble in the CPD and Stage III begins, as shown in Fig. 5. When the air plug leaves the capillary, it coalesces with the existing bubble in the CPD immediately [39-41], and forms a larger bubble inside the CPD, as shown in Fig. $5 b-e$, while the schematic illustration is shown in Fig. 5j. During the coalescence process, the surface area of the bubble is minimized, and surface energy is released. The extra energy will excite the pendant drop and cause it to vibrate slightly. The vibration of the pendant drop dissipates as time passes.

\subsubsection{Breakup of the compound pendant drop}

As more water slugs and air plugs enter the CPD alternatively, the accumulation

of fluids causes the CPD to grow both in weight and in size. The breakup of the CPD, as shown in Fig. 6, happens when the gravitational force is sufficiently large that the surface tension force cannot support the weight of the CPD. The neck of the CPD shrinks due to the pulling of the gravitational force, as illustrated in Fig. 6j. Plateau-Rayleigh instability [7] accelerates the breakup of the CPD, and a compound drop is formed, where the air bubble is fully engulfed by the water 
phase, as shown in Fig. 6i.

After the breakup of the CPD, the remaining liquid at the tip of the capillary recoils to form a new pendant drop of a small size. The stages of initialization, accumulation, coalescence, and breakup repeat as more air plugs and water slugs are introduced to the tip of the capillary.

\subsection{Formation and breakup of CPDs for air/SDS-solution system}

For the air/SDS-solution system, a significant difference from the air/water system is that the bubbles in CPDs do not coalesce. This is because the surfactant SDS reduces the air/water surface tension, and stabilizes the thin liquid film between bubbles by reducing film fluctuations [42].

Another difference between the air/water system and the air/ SDS-solution system is about the contact line of the liquid on the capillary during the formation and breakup of the CPDs. For the CPDs of the air/water system, the contact line is fixed at the outer circumference of the capillary tip, as shown in Figs. 3-6. In contrast, for the CPDs of the air/SDS-solution system, the contact line moves with time. After the breakup of the previous CPD, the contact line of the liquid climbs the capillary. This is because the surfactant increases the wettability of the liquid on the capillary. A simple measurement of contact angles confirmed that by adding SDS into DI water, the contact angle decreases from $61^{\circ}$ to $31^{\circ}$. The details to measure the contact angle on glass capillary is provided in Appendix A. With the accumulation of fluids in the CPD, the contact line moves downwards along the capillary until the CPD reaches a critical weight that the surface tension force cannot support, as shown in Fig. 7h. Then the neck of the CPD immediately shrinks off, and forms a compound drop with several bubbles in it, as shown in Fig. 7k.

Due to the movement of the contact line during the growth of the CPD, the bubble in the CPD may ascend to the vicinity of the contact due to the buoyancy, as shown in 
Fig. $7 \mathrm{~d}-\mathrm{g}$. Therefore, the shape of the CPD becomes asymmetric due to the presence of the bubbles around the capillary.

\subsection{Upstream velocity fluctuation caused by the breakup of compound pendant drops}

Fig. 8 illustrates the mechanisms of the breakup of pendant drops at the capillary tip affecting the upstream flow. As a pendant drop is formed at the tip, the Laplace pressure $\Delta p(t)$ depends on the size of the drop. Initially, the radius of the pendant drop is small,which results in a large Laplace pressure. As more fluids accumulates in the pendant drop (Stages II and III), the size of the pendant drop increases, and the Laplace pressure decreases gradually. This process continues until the breakup of the pendant drop, which results in a minimum pendant drop and a maximum Laplace pressure. The sudden increase in the Laplace pressure during the breakup will decelerate the flow in the capillary. The periodic breakup of pendant drops causes velocity fluctuations in the capillary. The fluctuated velocity in the capillary was recorded in the observing window, as shown in Fig. 8.

The velocity fluctuation in the capillary $u(t)$ was calculated from the sequences of images using Eq. (1). Fig. 10 shows the variation of the normalized velocity with time in the upstream at different flow rates. The normalized velocity is defined as

$$
\hat{u}(t) \equiv \frac{u(t)}{U}
$$

where $U$ is the time-average velocity, which is defined as

$U \equiv \frac{1}{T} \int_{0}^{T} u(t) d t$

where $\mathrm{T}$ is the selected time period that $U$ remains unchanged. In the analysis, $T$ is selected about four periods of the fluctuation, as illustrated in Fig. 9. After normalization, $\hat{u}(t)$ preserves the feature of the velocity fluctuation, and gives a clear view of the relative magnitude of the velocity fluctuation. 
The volume fractions of air at different flow rates of DI water were measured from the images of the air-plug/water-slug flow in the capillary as follows

$f_{\text {air }} \equiv \frac{L_{\text {air-plug }}}{L_{\text {air-plug }}+L_{\text {liquid-slug }}}$

where $L_{\text {air-plug }}$ and $L_{\text {water-slug }}$ are the lengths of the air plug and the liquid slug in the images. For the air/water system, when the flow rate of DI water increases from 0.6 $\mathrm{ml} / \mathrm{min}$ to $0.9 \mathrm{ml} / \mathrm{min}$, the volume fraction of air decreases from 0.42 to 0.32 .

The velocity fluctuation of air/water system is shown in Fig. 10. With the increase in the flow rate, the magnitude of the fluctuation of the normalized velocity decreases. This is due to the fact that with the increase in the flow rate, the time average velocity $U$ increases proportionally. But the size of the pendant drop remains almost unchanged, which causes the Laplace pressure within the pendant drop nearly unchanged. Therefore, the fluctuation of the normalized velocity $\hat{u}$ decreases.

Fig. 11 shows the variation of normalized velocity for the air/ SDS-solution systems. By adding the surfactant into DI water, the surface tension is reduced from $72.8 \mathrm{mN} / \mathrm{m}$ to $36.16 \mathrm{mN} / \mathrm{m}$, as shown in Table 1 . Because the Laplace pressure is proportional to the surface tension, a smaller surface tension results in a small Laplace pressure, and a small magnitude of velocity fluctuation. Another reason of reducing the velocity fluctuation by the surfactant may be the movement of the contact line for the air/ SDSsolution system as discussed in Section 3.2. For the air/water system, the contact line is fixed at the outer circumference during the formation and the breakup of the CPDs. After the pinch-off of the previous CPD, a new small CPD results in a large Laplace pressure and a large magnitude of velocity fluctuation. In contrast, for the air/SDSsolution system, the surfactant reduces the contact angle of the liquid on the glass capillary. The contact line moves upwards along the capillary when the CPD is small. The upward movement of the contact line results that the CPD engulfs the capillary tip, 
which increases the visual volume of the CPD (i.e., the volume wrapped by the surface of the CPD) and decreases the curvature. Therefore, the movement of the contact line may reduce the variation of the Laplace pressure and reduces the velocity fluctuation in the upstream.

\subsection{Size distribution of the compound drops}

The size of the compound drops produced by the breakup of CPDs depends on two breakup events, (i) the generation of bubbles in the flow focusing geometry in the microchannel, and (ii) the breakup of CPDs at the tip of the glass capillary. In the microchannel, the flow focusing geometry has the capability to produce monodisperse bubbles when working in the squeezing or dripping regimes [37,38], during which the capillary number is low. When the capillary number is sufficiently high, breakup of bubbles happens in the jetting regime, during which the bubbles become poly-disperse. The second breakup event, the breakup at the capillary tip, may occur in the dripping or jetting regimes depending on the Weber number $W e$, which characterizes the relative effect between the inertial force and the surface tension force. Dripping of drops at the capillary tip happens when the Weber number $W e$ is small. In the dripping regime, the drops produced at the capillary tip are monodisperse. Therefore, the system used in this study has the potential to produce monodisperse compound drops when (i) the breakup of bubbles at the flow focusing geometry is in the squeezing or the dripping regime, and (ii) the breakup of CPDs at the capillary tip is in the dripping regime.

To characterize the size distribution of the compound drops produced by breakup of the CPDs, the sizes of the compound drops were measured from the high speed images using a customized MATLAB program, i.e., $A_{\text {pixel, }}$ the area of the compound drops in images by pixel. The histogram of $A_{\text {pixel }}$ was plotted in Fig. 12a. The histogram of $A_{\text {pixel }}$ shows that the size of the compound drops are monodisperse with a polydispersity of $\zeta_{A_{\text {pixel }}}=8.5 \%$, which is defined as the standard deviation of $A_{\text {pixel }}$ divided by the average value of $A_{\text {pixel }}$. 
Because there are two immiscible fluids (liquid and gas) in each compound drops, the consistency of the liquid volumes in different compound drops is important to ensure the monodispersity of the compound drops. Since liquid was introduced into the microchannel at a constant flow rate, the volume of liquid in a compound drop could be calculated from the time interval between two consecutive breakup events at the capillary tip $\Delta T$.

$V_{\text {liquid }}=Q \Delta T$

The time interval $\Delta T$ was determined by counting the frames of the high speed images (500 fps) between two consecutive breakup events at the capillary tip. The histogram of $V_{\text {liquid }}$ was plotted in Fig. 12b, which shows that the liquid volumes in different compound drops are monodisperse. The polydispersity of $V_{\text {liquid }}$ is $\zeta_{V_{\text {liquid }}}=6.9 \%$, which is defined as the standard deviation of $V_{\text {liquid }}$ divided by the average volume of $V_{\text {liquid. }}$

\section{Conclusions}

In this study, we investigated the formation and breakup of compound pendant drops. Four typical stages for the air/water system have been identified: (1) initialization of a small compound pendant drop, (2) accumulation of liquid in the compound pendant drop, (3) coalescence of bubble with the subsequent air plug, and finally (4) breakup of the compound pendant drop. These four stages of initialization, accumulation, coalescence, and breakup repeat as more air plugs and water slugs are introduced to the tip of the capillary. For the air/SDS-solution system, due to the presence of the surfactant, the bubbles in the CPDs do not coalesce, and the contact lines of the CPDs initially climb along the capillary and then move downwards with the increases in the weight of the CPDs. The breakup of the CPDs results in compound drops with several bubbles within them. At the capillary tip, the formation and breakup of the compound pendant drops causes velocity fluctuation in the upstream. The velocity fluctuation reduces by adding 
surfactant into the liquid. The size distribution of the compound drops produced by the breakup of CPDs is affected by the breakup event at the flow focusing geometry and the breakup event at the capillary tip. The current system is able to produce monodisperse compound drops. The polydispersity of the compound drops in this experiment was less than $10 \%$.

\section{Appendix A. Measurement of contact angles on glass capillary}

To quantitatively measure the contact angle of DI water or the SDS solution on the glass capillary, we used a glass slide $(75 \mathrm{~mm} \times 50 \mathrm{~mm})$ and spread the water or the SDS solution on it, as shown in Fig. A.1. The liquid is sufficient to result in a flat surface of liquid. Subsequently, the glass capillary was dipped vertically into the liquid, and observed horizontally from the side using a CCD camera. Fig. A.2a$\mathrm{b}$ are the images obtained from the CCD camera for DI water and for the SDS solution, respectively. From the images, we can confirm that adding the surfactant SDS into DI water increases the wettability of the liquid on the glass capillary. The contact angle decreases from $61^{\circ}$ to $31^{\circ}$ by adding surfactant SDS into DI water. 


\section{References}

[1] R. Badie, D.F. d. Lange, Mechanism of drop constriction in a drop-on-demand inkjet system, Proc. Math Phys. Eng. Sci. 453 (1997) 2573-2581.

[2] J. Eggers, Nonlinear dynamics and breakup of free-surface flows, Rev. Mod. Phys. 69 (1997) 865.

[3] J. Eggers, E. Villermaux, Physics of liquid jets, Rep. Prog. Phys. 71 (2008) 036601.

[4] B. Ambravaneswaran, H.J. Subramani, S.D. Phillips, O.A. Basaran, Drippingjetting transitions in a dripping faucet, Phys. Rev. Lett. 93 (2004) 034501.

[5] S.D.R. Wilson, The slow dripping of a viscous fluid, J. Fluid Mech. 190 (1988) 561-570.

[6] X. Zhang, Dynamics of growth and breakup of viscous pendant drops into air, J. Colloid Interf. Sci. 212 (1999) 107-122.

[7] D.H. Michael, Meniscus stability, Annu. Rev. Fluid. Mech. 13 (1981) 189-216.

[8] X.D. Shi, M.P. Brenner, S.R. Nagel, A cascade of structure in a drop falling from a faucet, Science 265 (1994) 219-222.

[9] J.C. Burton, P. Taborek, Two-dimensional inviscid pinch-off: An example of selfsimilarity of the second kind, Phys. Fluids 19 (2007) 102109.

[10] B. Ambravaneswaran, S.D. Phillips, O.A. Basaran, Theoretical analysis of a dripping faucet, Phys. Rev. Lett. 85 (2000) 5332.

[11] S. Middleman, Modeling Axisymmetric Flows: Dynamics of Films, Jets, and Drops, Academic Press, San Diego, 1995.

[12] D.F. Zhang, H.A. Stone, Drop formation in viscous flows at a vertical capillary tube, Phys. Fluids 9 (1997) 2234-2242.

[13] Y.C. Liao, E.I. Franses, O.A. Basaran, Deformation and breakup of a stretching liquid bridge covered with an insoluble surfactant monolayer, Phys. Fluids 18 (2006) 022101.

[14] Q. Xu, Y.-C. Liao, O.A. Basaran, Can surfactant be present at pinch-off of a liquid filament?, Phys Rev. Lett. 98 (2007) 054503.

[15] M.A. Rother, R.H. Davis, Buoyancy-driven breakup of an isolated drop with surfactant, Phys. Rev. Lett. 101 (2008) 044501. 
[16] R. Suryo, O.A. Basaran, Dripping of a liquid from a tube in the absence of gravity, Phys. Rev. Lett. 96 (2006) 034504.

[17] X. Zhang, O.A. Basaran, Dynamics of drop formation from a capillary in the presence of an electric field, J. Fluid. Mech. 326 (1996) 239-263.

[18] R.T. Collins, J.J. Jones, M.T. Harris, O.A. Basaran, Electrohydrodynamic tip streaming and emission of charged drops from liquid cones, Nat. Phys. 4 (2008) 149-154.

[19] R.E. Johnson, S.S. Sadhal, Fluid mechanics of compound multiphase drops and bubbles, Annu. Rev. Fluid. Mech. 17 (1985) 289-320.

[20] S. Tasoglu, G. Kaynak, A.J. Szeri, U. Demirci, M. Muradoglu, Impact of a compound droplet on a flat surface: A model for single cell epitaxy, Phys. Fluids 22 (2010) 082103.

[21] A. Borhan, H. Haj-Hariri, A. Nadim, Effect of surfactants on the thermocapillary migration of a concentric compound drop, J. Colloid Interf. Sci. 149 (1992) 553560.

[22] L. Rosenfeld, O.M. Lavrenteva, A. Nir, On the thermocapillary motion of partially engulfed compound drops, J. Fluid. Mech. 626 (2009) 263-289.

[23] A.S. Utada, E. Lorenceau, D.R. Link, P.D. Kaplan, H.A. Stone, D.A. Weitz, Monodisperse double emulsions generated from a microcapillary device, Science 308 (2005) 537-541.

[24] S. Okushima, T. Nisisako, T. Torii, T. Higuchi, Controlled production of monodisperse double emulsions by two-step droplet breakup in microfluidic devices, Langmuir 20 (2004) 9905-9908.

[25] D. Dendukuri, P.S. Doyle, The synthesis and assembly of polymeric microparticles using microfluidics, Adv. Mater. 21 (2009) 4071-4086.

[26] J.I. Park, A. Saffari, S. Kumar, A. Günther, E. Kumacheva, Microfluidic synthesis of polymer and inorganic particulate materials, Ann. Rev. Mater. Res. 40 (2010) 415-443.

[27] A.J. deMello, Control and detection of chemical reactions in microfluidic systems, Nature 442 (2006) 394-402.

[28] D. Janasek, J. Franzke, A. Manz, Scaling and the design of miniaturized chemical- 
analysis systems, Nature 442 (2006) 374-380.

[29] H. Song, J.D. Tice, R.F. Ismagilov, A microfluidic system for controlling reaction networks in time, Angew. Chem. - Int. Edit. 42 (2003) 768-772.

[30] X. Niu, Y.K. Lee, Efficient spatial-temporal chaotic mixing in microchannels, J. Micromech. Microeng. 13 (2003) 454-462.

[31] N.T. Nguyen, Z. Wu, Micromixers - a review, J. Micromech. Microeng. 15 (2005) R1-R16.

[32] Y. Ma, C.P. Sun, M. Fields, Y. Li, D.A. Haake, B.M. Churchill, C.M. Ho, An unsteady microfluidic t-form mixer perturbed by hydrodynamic pressure, J. Micromech. Microeng. 18 (2008) 045015.

[33] J.M. Ottino, The Kinematics of Mixing: Stretching Chaos, and Transport, Cambridge University Press, New York, 1989.

[34] H. Aref, The development of chaotic advection, Phys. Fluids 14 (2002) 13151325.

[35] V. van Steijn, M.T. Kreutzer, C.R. Kleijn, Velocity fluctuations of segmented flow in microchannels, Chem. Eng. J. 135 (2007) S159-S165.

[36] N.R. Beer, K.A. Rose, I.M. Kennedy, Observed velocity fluctuations in monodisperse droplet generators, Lab Chip 9 (2009) 838-840.

[37] S.L. Anna, N. Bontoux, H.A. Stone, Formation of dispersions using "flow focusing" in microchannels, Appl. Phys. Lett. 82 (2003) 364-366.

[38] G.F. Christopher, S.L. Anna, Microfluidic methods for generating continuous droplet streams, J. Phys. D. Appl. Phys. 40 (2007) R319-R336.

[39] D.G.A.L. Aarts, H.N.W. Lekkerkerker, H. Guo, G.H. Wegdam, D. Bonn, Hydrodynamics of droplet coalescence, Phys. Rev. Lett. 95 (2005) 164503.

[40] D.G.A.L. Aarts, H.N.W. Lekkerkerker, Droplet coalescence: drainage, film rupture and neck growth in ultralow interfacial tension systems, J. Fluid Mech. 606 (2008) 275-294.

[41] D.Y.C. Chan, E. Klaseboer, R. Manica, Film drainage and coalescence between deformable drops and bubbles, Soft Matter 7 (2011) 2235-2264.

[42] T. Tadros, Applied Surfactants: Principles and Applications, Wiley-VCH, Weinheim, 2005. 


\section{List of Tables}

Table $1 \quad$ Properties of the fluids at $20^{\circ} \mathrm{C}$. 


\section{List of Figures}

Fig. 1. $\quad$ Experimental setup: (a) Schematic diagram of the experimental setup;

(b) Schematic diagram of the air bubble formation in the microchannel;

(c) Schematic diagram of the air-plug/water-slug flow in the capillary and the compound pendant drop at the tip of the capillary; (d) Schematic diagram of the connection between the PMMA channel and the glass capillary. (e) Image of the microchannel with a glass capillary connected to the outlet of the microchannel.

Fig. 2. Procedure of image processing to obtain the velocity from the experimental video: (a) A typical frame of the video; (b) Cropping the region of the capillary for further analysis; (c) The background image obtained by averaging many frames; (d) Subtracting the background image from each frame to obtain the bubbles in the images; (e) Converting the grayscale images to black-and-white binary images to get a clear bubble; (f) Averaging the image pixels by column to get the profile; (g) Taking the gradient of the profile to find out the location of the fronts and rears of the bubbles; (h) Calculating the velocities of the front and rear of the bubble by comparing two successive frames. Averaging the velocities of the fronts and the rears of the air plugs and take the average $u\left(t_{k}\right)$ as the velocity of the fluids at that time $\mathrm{t}_{\mathrm{k}}$.

Fig. 3. Initialization of a small compound pendant drop by entering an air plug into a small pendant drop: (a-i) Sequence of images; and (j) Schematic illustration.

Fig. 4. Accumulation of liquid in the compound pendant drop: (a-f) Sequence of images and (g) Schematic illustration. 
Fig. 5. Coalescence of the bubble in the CPD with the subsequent air plug: (a-i) Sequence of images; and (j) Schematic illustration.

Fig. 6. Breakup of the CPD: (a-i) Sequence of images; and (j) Schematic illustration.

Fig. 7. Image sequence of formation and breakup of CPDs for the air/SDSsolution system.

Fig. 8. Schematic diagram of the upstream velocity fluctuation caused by the breakup: (a) The large radius of the CPD produces a low curvature, a low Laplace pressure, and a low pressure inside the capillary. (b) The small size of the CPD after the breakup produces a high curvature, a large Laplace pressure, and a large pressure inside the capillary. The sudden increases in the curvature will increase the pressure in the capillary (from $p\left(t_{1}\right)$ to $p\left(t_{2}\right)$ ) and decelerate the flow in the capillary (from $u\left(t_{1}\right)$ to $\left.u\left(t_{2}\right)\right)$. The velocity fluctuation was recorded at the observing window.

Fig. 9 The velocity of the fluid $u(t)$ is normalized by $U$, which is the average velocity of the fluid over interval T. (a) Absolute velocity of the fluid $u(t)$; (b) Normalized velocity of the fluid $\hat{u}(t))$.

Fig. 10. Velocity fluctuations in the capillary for CPDs of the air/water system at different flow rates of DI water. The flow rates are (a) $0.6 \mathrm{ml} / \mathrm{min}$, (b) $0.7 \mathrm{ml} / \mathrm{min}$, (c) $0.8 \mathrm{ml} / \mathrm{min}$, and (d) $0.9 \mathrm{ml} / \mathrm{min}$, respectively. The superficial velocities of the water stream are $5.1,5.9,6.8$, and $7.6 \mathrm{~cm} / \mathrm{s}$, respectively, and the volume fractions of air $f_{\text {air }}$ are 0.42, 0.40, 0.33, and 0.32 , respectively.

Fig. 11. Velocity fluctuations in the capillary for CPDs of the air/SDS-solution 
system at different flow rates of the SDS solution. The flow rates are (a) $0.6 \mathrm{ml} / \mathrm{min}$, and (b) $0.9 \mathrm{ml} / \mathrm{min}$, respectively. The superficial velocities of the SDS solution are 5.1 and $7.6 \mathrm{~cm} / \mathrm{s}$, respectively, and the volume fractions of air $f_{\text {air }}$ are 0.57 and 0.49 , respectively.

Fig. 12. Size distribution of the compound drops produced by breakup of CPDs at the capillary tip. The liquid is the SDS solution (2\% by weight in water), and its flow rate is $0.6 \mathrm{ml} / \mathrm{min}$. (a) Histogram of the area of the compound drops $A_{\text {pixel }}$ measured from high speed images. The polydispersity of $A_{\text {pixel }}$ is $\zeta_{A_{\text {pixel }}}=8.5 \%$. (b) Histogram of the liquid volume $V_{\text {liquid }}$ determined by counting the frames of the high speed images (500 fps) between two consecutive breakup events at the capillary tip. The polydispersity of $V_{\text {liquid }}$ is $\zeta_{V_{\text {liquid }}}=6.9 \%$.

Fig. A.1. Schematic diagram of measuring the contact angles of DI water and the SDS solution on the wall of the glass capillary.

Fig. A.2. Images for contact angle measurement on the glass capillary. (a) The image of contact angle for DI water on the glass capillary. The contact angle for DI water is $61^{\circ}$. (b) The image of contact angle for the SDS solution on the glass capillary. The contact angle for the SDS solution is $31^{\circ}$. 


\begin{tabular}{llll}
\hline & Air & Water & SDS solution \\
\hline Density $\left(\mathrm{kg} / \mathrm{m}^{3}\right)$ & 1.204 & 997 & 997 \\
Viscosity $(\mathrm{Pa} \cdot \mathrm{s})$ & $1.84 \times 10^{-5}$ & $1 \times 10^{-3}$ & $1 \times 10^{-3}$ \\
Surface tension $(\mathrm{mN} / \mathrm{m})$ & & 72.8 & 36.16 \\
\hline
\end{tabular}

Table 1 
Water column for

constant pressure

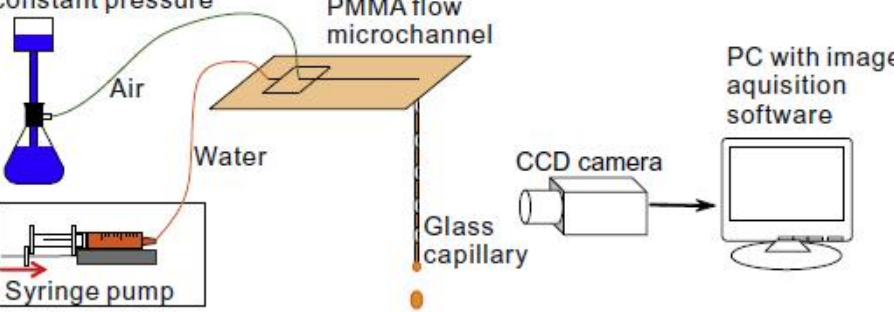

(a)

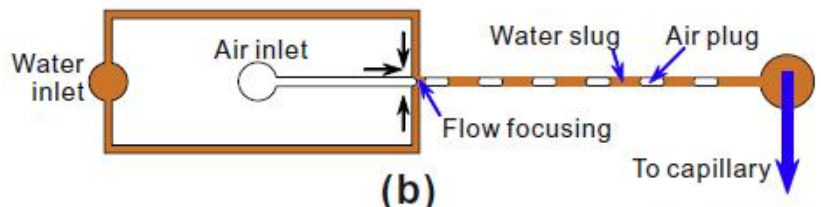

(b)

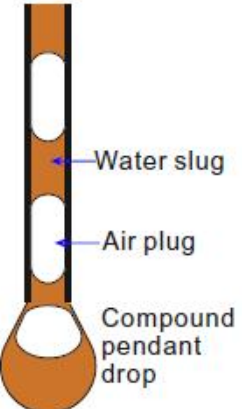

(c)

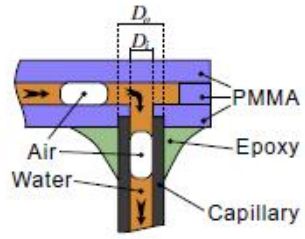

(d)

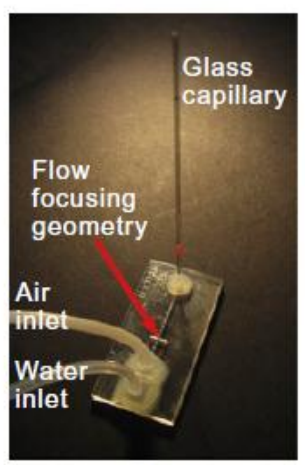

(e)

Fig. 1 
(a)

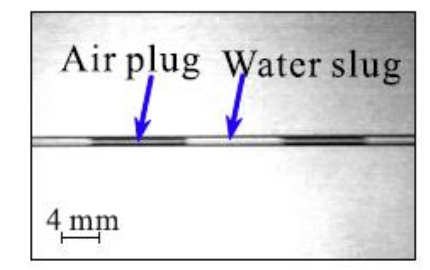

(b)

(c)

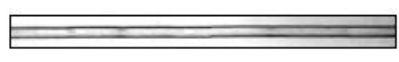

(d)

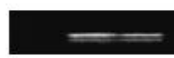

(e)

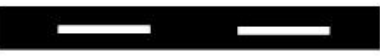

(f)

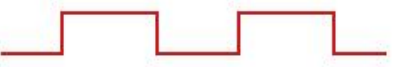

(g) 1

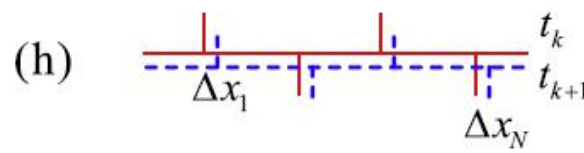

Fig. 2 

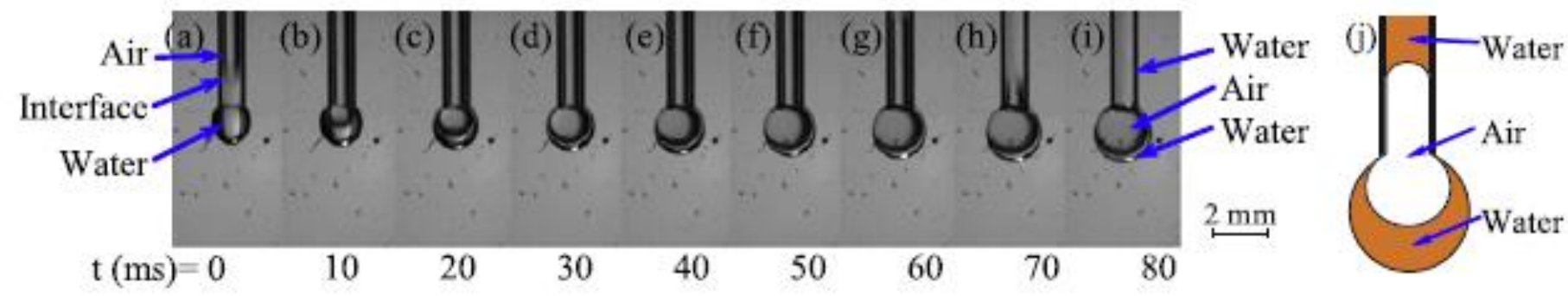

Fig. 3 

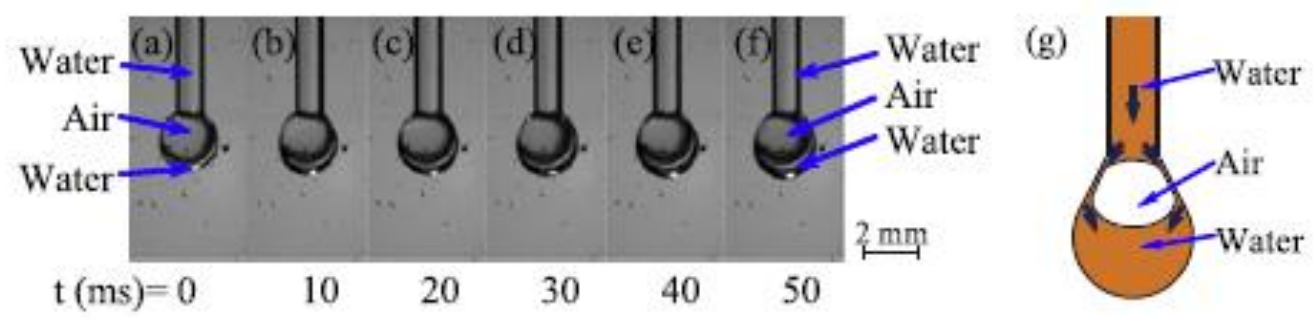

Fig. 4 


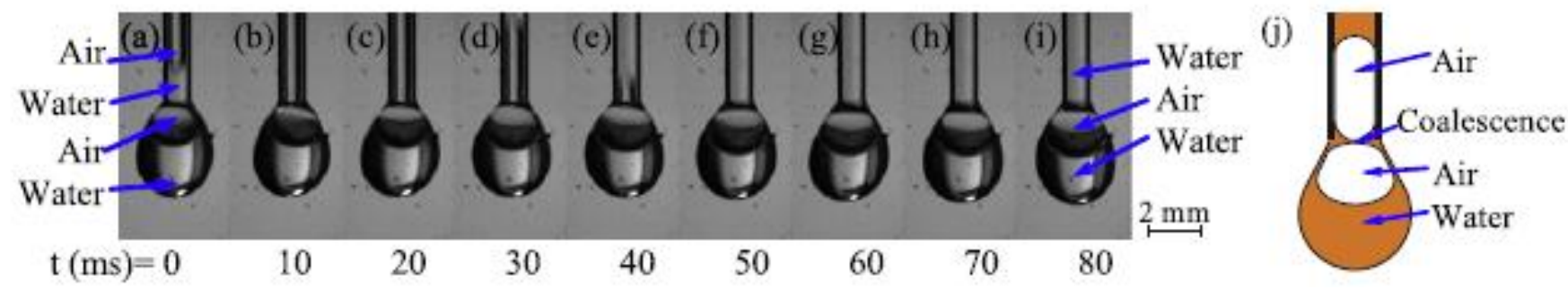

Fig. 5 

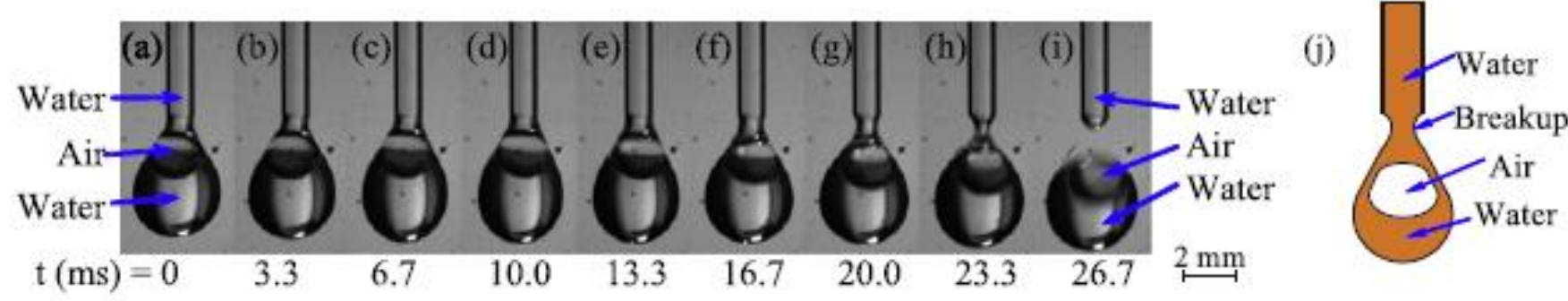

Fig. 6 


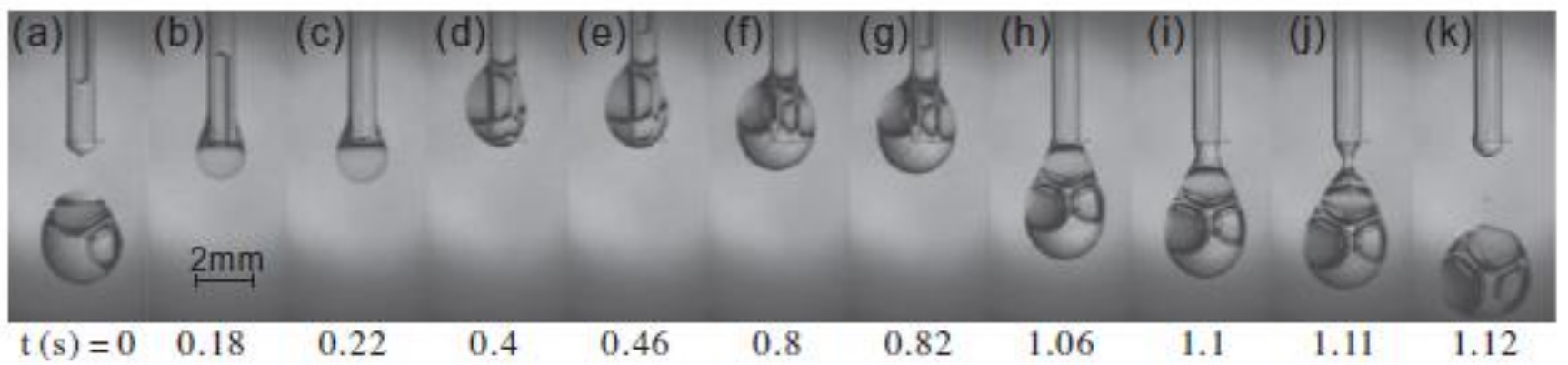

Fig. 7 


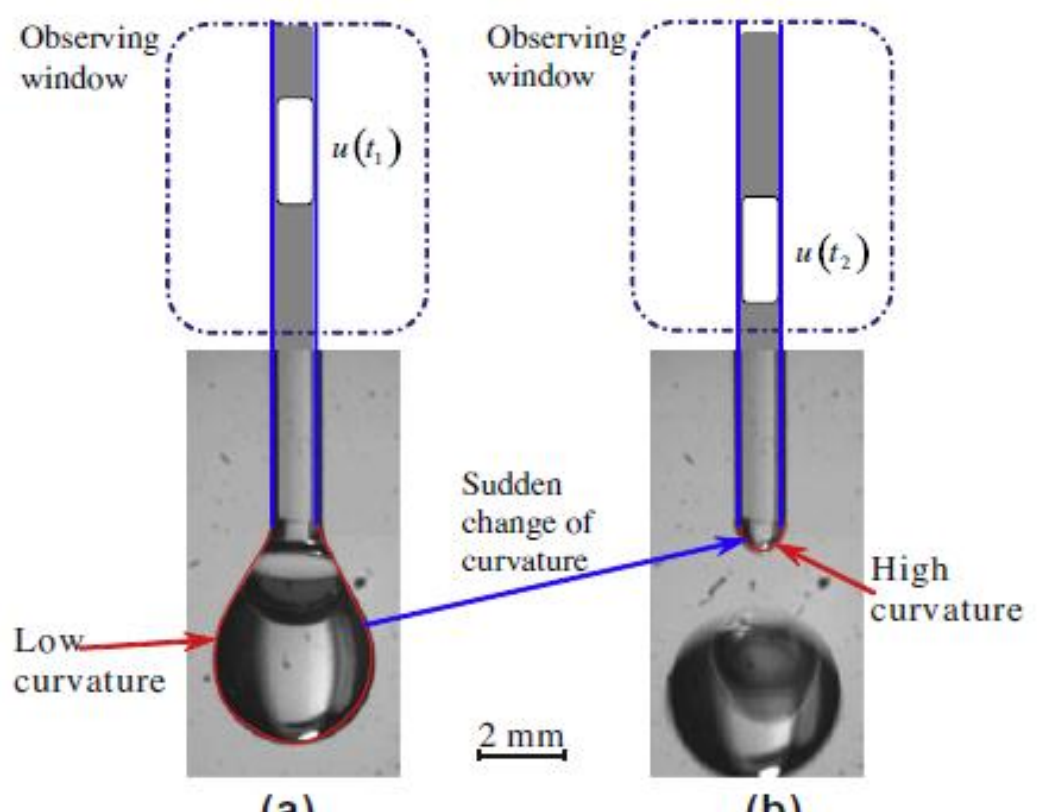

(a)

(b)

Fig. 8 


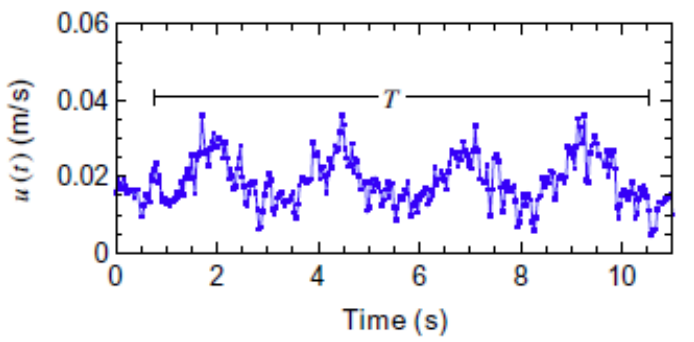

(a)

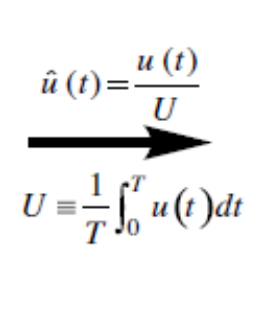

Fig. 9

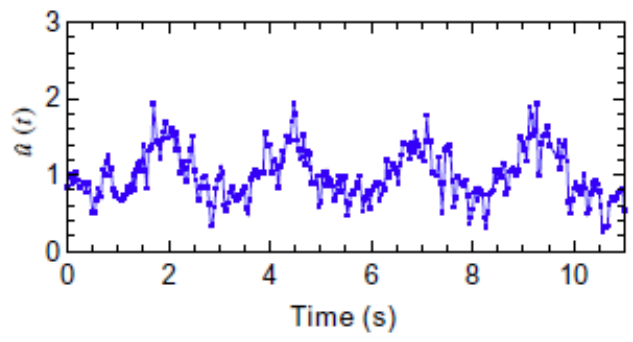

(b) 


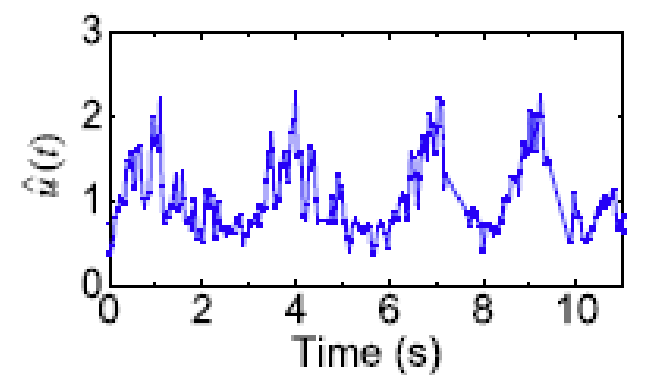

(a)

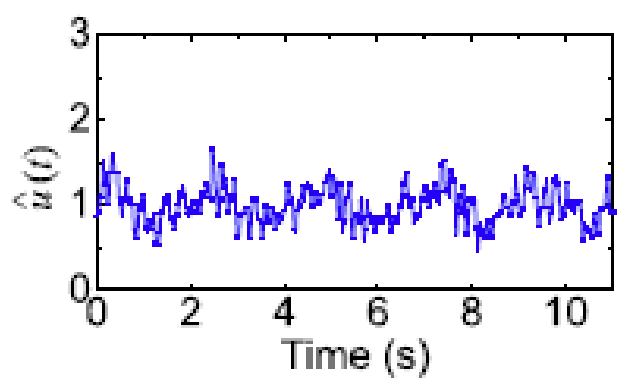

(c)

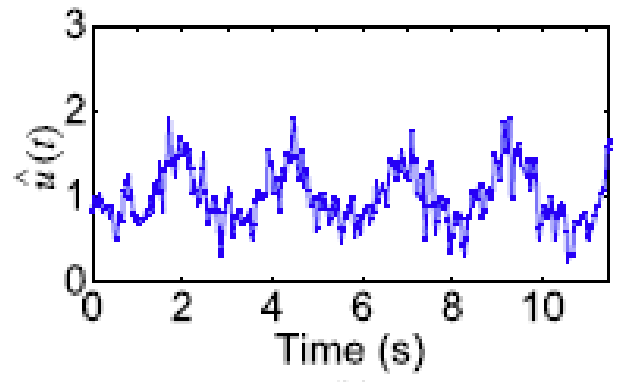

(b)

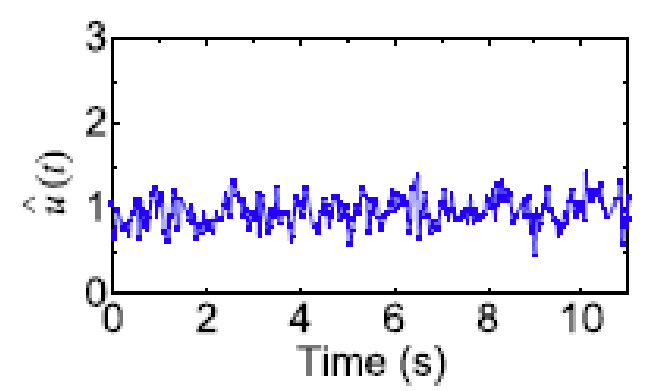

(d)

Fig. 10 


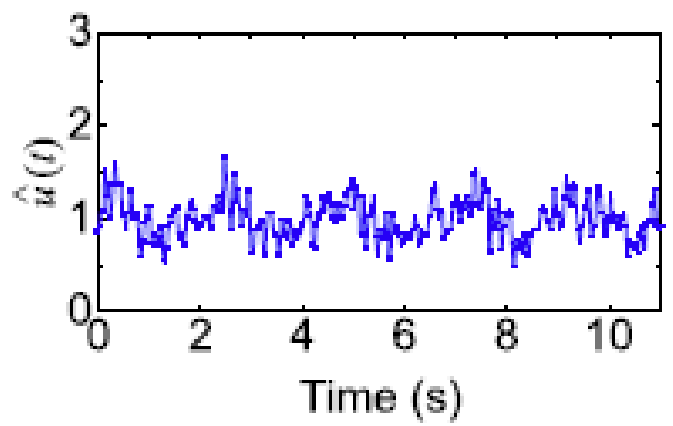

(a)

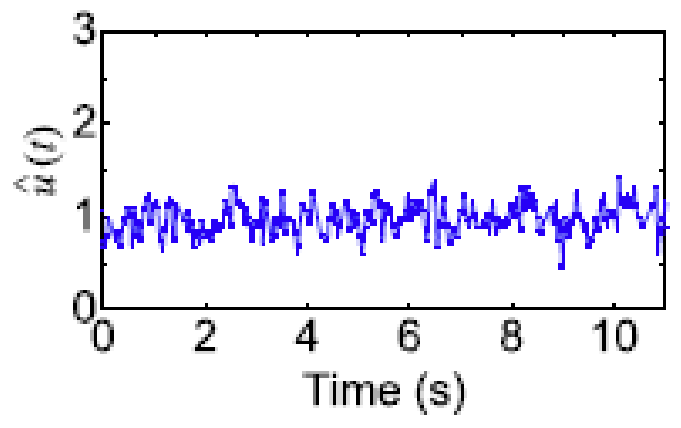

(b)

Fig. 11 


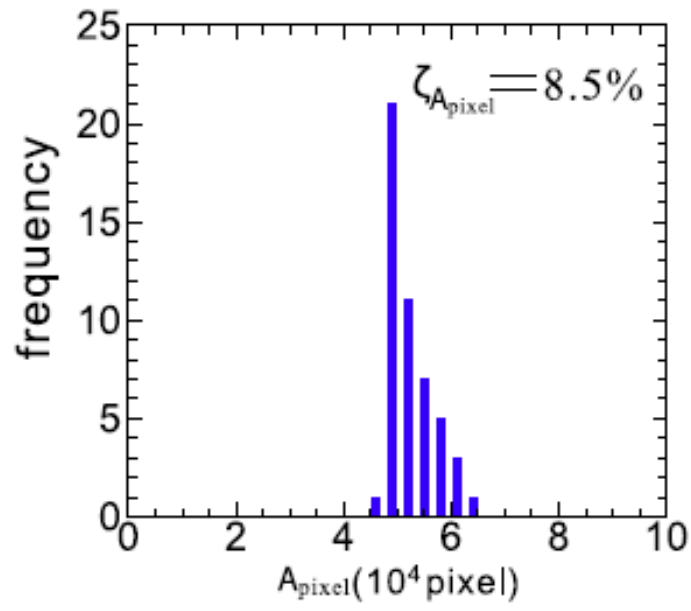

(a)

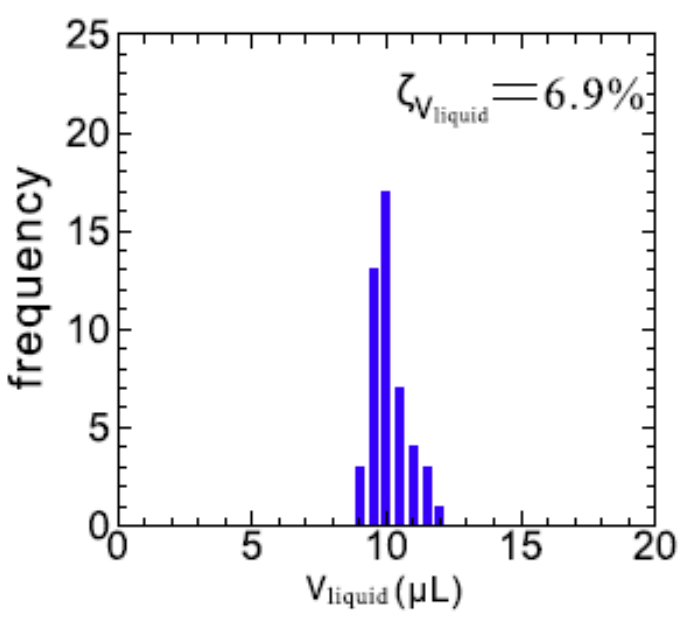

(b)

Fig. 12 


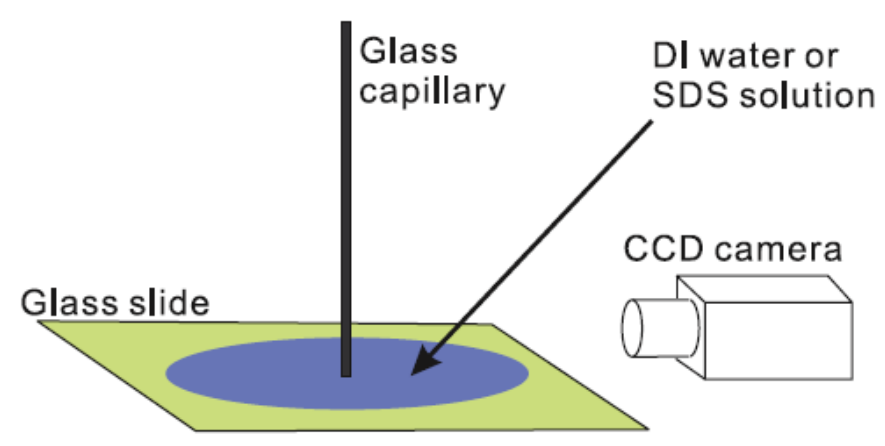

Fig. A.1. 


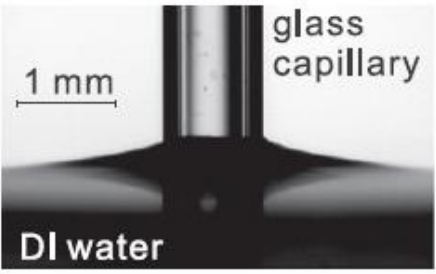

(a)

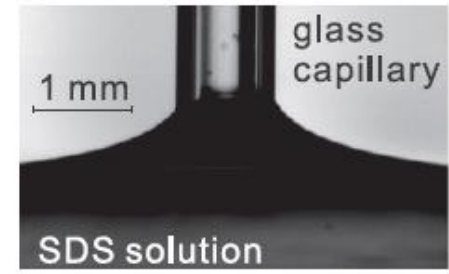

(b)

Fig. A.2 